\title{
On application of algebra of quaternions II
}

\author{
Emran Sasi Althabit*, Ismail.M.Mohamed ${ }^{* *}$ \\ ${ }^{*}$ Higher Institute for water Affairs
${ }^{* *}$ Academic of Engineering and Medical Sciences \\ DOI: 10.29322/IJSRP.11.02.2021.p11041 \\ http://dx.doi.org/10.29322/IJSRP.11.02.2021.p11041
}

\begin{abstract}
We introduced algebraic formulations of time as quaternions, Clifford Algebras, we deduce the Lorentz transformation from the Minkowski Space.
\end{abstract}

Index Terms- Clifford Algebras, K-algebra, multivector, Affine vector space, Minkowski Space.

\section{Algebraic Formulations OF TIME}

$\mathrm{I}^{\mathrm{n}}$ order to proceed with our approach, we now wish to algebraically model three- dimensional physical space. The distinct approaches are commonly utilized to model various physical systems such as three-vectors,four-vectors, matrices, complex numbers, quaternions and complex spinors, depending on the specific application. This fact is indeed quite surprising, in that, one of the specific goals of nineteenth century science was to find the correct algebra for physical space [6]. The objective was initially led by Hamiltonn who produced the quaternion algebra through generalizing the two-dimensional complex numbers to three dimensions. Indeed, due to the general success of complex numbers in algebraically describing the properties of the plane Hamilton reasoned that quaternions should therefore properly describe the algebra of three-dimensional space. This then lead to the first attempt at a rigorous mathematical definition of a unified space and time.Hamilton wrote the quaternion as $q=t+x_{1} i+$ $x_{2} j+x_{3} k \quad(1.1)$ where $x_{1}, x_{2}, x_{3} \in \mathbb{R}^{3}$ and the three basis vectors are subject to the well known quaternionic relations $i^{2}=$ $j^{2}=k^{2}=-1$

Hamilton's quaternions form a 4-D associative normed division algebra over the real numbers represented by $\mathbb{H}$. Hamilton defined $\vec{x}=x_{1} i+x_{2} j+x_{3} k$ as a vector quaternion to take the role of Cartesian vectors in order to describe spatial vectors, and then also proposed, around 50 years before Minkowski, that if the scalar " $t$ " was identified with time then the quaternion can be used as a representation for a unified four-dimensional spacetime. Hamilton stating "Time is said to have only one dimension, and space to have three dimensions. ...

The mathematical quaternion partakes of both these elements; it may be said to be 'time plus space', or 'space plus time': and in this sense it has,or at least involves a reference to, four dimensions". Indeed squaring the quaternion we find $q^{2}=t^{2}-x^{2}+2 t x \quad$ (1.2) the scalar component $t^{2}-x^{2}$ thus producing the invariant spacetime distance.

Minkowski, after indeed considering the quaternions, but viewing them as too restrictive for describing spacetime, chose rather to extend the Gibbs-Heaviside thre-vector system with the addition of a time coordinate to create a four-component vector producing the modern description of spacetime, as a fourvector

$X=[t, \vec{x}] \quad$ (1.3)

Defining a involution $X^{*}=[t,-\vec{x}]$ we then

produce the invariant distance

$X X^{*}=[t, \vec{x}][t,-\vec{x}]=t^{2}-x^{2}$

Comparing these two descriptions we can see that they provide some significant differences. To begin with, Hamilton's description views time as an intrinsic part of the description of three-dimensional space. On the other hand, with the Minkowski formulation, the immed-iate implication is that time is an additional Euclidean-type dimension. Although this assu-mption is qualified by the fact that time contributes an opposite sign to the metric distance and so distinct from a regular four dimensional Cartesian vector. Note that the octonions, being the generalization of quaternions, have also been considered as an expanded arena for spacetime.

It is an historical fact that the vector quaternions were found difficult to work with and not suitable to describe Cartesian vectors and were replaced by the Gibbs vector system in use today [6]. The reason for Hamilton's failed attempt to algebraically describe three-dimensional space, is that by generalizing the complex numbers of the plane to three dimensions he actually only produced the rotational algebra for three dimensions.

To properly describe three-dimensional space we further need to generalize the quaternions to include a true Cartesian vector component [13]. This generalization of quaternions was in fact achieved by Clifford with the eight dimensional Clifford algebra over three dimensions of $C \ell\left(\mathbb{R}^{3}\right)[14]$

\section{Clifford Algebras}

Action the group on quaternions(2-1)

We define an action of the group of unit quaternions, $S U(2)$, on $\mathbb{R}^{3}$. For this, we use the fact that $\mathbb{R}^{3}$ can be identified with the pure quaternions in $\mathbb{H}$, namely, the quaternions of the form $\vec{x}=x_{1} i+$ $x_{2} j+x_{3} k$, where $\left(x_{1}, x_{2}, x_{3}\right) \in \mathbb{R}^{3}$. Then, we define the action of $S U(2)$ over $\mathbb{R}^{3}$ by $Z \cdot X=Z X Z^{-1}=Z X Z^{*}$

where $Z \in S U(2)$ and $X$ is any pure quaternion. [9]

Now, it turns out that the map $\rho Z$ where

$\rho z(X)=Z X Z^{-1}$ is indeed a rotation, and that the map $\rho: Z \mapsto \rho z$ is a surjective homomorphism, $\rho: S U(2) \rightarrow S O(3)$, whose kernel is $\{-1,1\}$, where 1 denotes the multiplicative unit quaternion.

We can also define an action of the group $S U(2) \times S U(2)$ over $\mathbb{R}^{4}$ by identifying $\mathbb{R}^{4}$ with the quaternions. In this case,

$(Y, Z) \cdot X=Y X Z^{*}$

(2.2) 
where $(Y, Z) \in S U(2) \times S U(2)$ and $X \in \mathbb{H}$ is any quaternion. Then, the map $\rho_{Y, Z^{*}}$ is a rotation where $\rho_{Y, Z^{*}}(X)=Y X Z^{*}$, and the map $\rho:(Y, Z) \mapsto \rho_{Y, Z^{*}}$ is a surjective homomorphis, $\rho: S U(2) \times$ $S U(2) \rightarrow S O(4)$, whose kernel is $\{(1,1),(-1,-1)\}$.

Thus, we observe that for $n=2,3,4$, the rotations in $S O(n)$ can be realized via the linear action of some group (the case $n=1$ is trivial, since $S O(1)=\{1,-1\}$. It is also the case that the action of each group can be somehow be described in terms of multiplication in some larger algebra "containing" the original vector space $\mathbb{R}^{n}$ ( $\mathbb{C}$ for $n=2, \mathbb{H}$ for $n=3,4$ ). However, these groups appear to have been discovered in an ad hoc fashion, and there does not appear to be any universal way to define the action of these groups on $\mathbb{R}^{n}$. It would certainly be nice if the action was always of the form $Z \cdot X=Z X Z^{-1}\left(=Z X Z^{*}\right)$

A systematic way of constructing groups realizing rotations in terms of linear action, using a uniform notion of action, does exist. Such groups are the spinor groups, to be described in the following paragraphs.

We explained in Paragraph.1how the rotations in $S O(3)$ can be realized by the linear action of the group of unit quaternions, $S U(2)$, on $\mathbb{R}^{3}$, and how the rotations in $S O(4)$ can be realized by the linear action of the group $S U(2) \times S U(2)$ on $\mathbb{R}^{4}$.The main reasons why the rotations in $\mathrm{SO}(3)$ can be represented by unit quaternions are the following:

(1) For every nonzero vector $\vec{u} \in \mathbb{R}^{3}$, the reflection $s_{\vec{u}}$ about the hyperplane Perpendi-cular to $\vec{u}$ is represented by the map $v \mapsto$ -uvu $u^{-1} \quad$ (2.3) here $\vec{u}$ and $\vec{v}$ are viewed as pure quaternions in $\mathbb{H}$ (i.e., if $\vec{u}=\left(u_{1}, u_{2}, u_{3}\right)$, then view

$\vec{u}$, as $u_{1} i+u_{2} j+u_{3} k$, and similarly for $\vec{v}$ ).

(2) The group $\mathrm{SO}(3)$ is generated by the reflection.

The group of unit quaternions $S U(2)$ turns out to be isomorphic to the spinor group

Spin(3). Because Spin(3) acts directly on $\mathbb{R}^{3}$,

the representation of rotations in $S O$ (3) by elements of Spin(3) may be viewed as more natural than the representation by unit quaternions. The group $\mathrm{S} U(2) \times S U(2)$ turns out to be isomorphic to the spinor group $\operatorname{Spin}(4)$, but this isomorphism is less obvious. In summary, we are going to define a group $\operatorname{Spin}(n)$ representing the rotations in $S O(n)$, for any $\geq 1$, in the sense that there is a linear action of $\operatorname{Spin}(n)$ on $\mathbb{R}^{n}$ which induces a surjective homomorphism, $\rho: \operatorname{Spin}(n) \rightarrow S O(n)$, whose kernel is $\{-1,1\} 1 \mathrm{~g}$. Furthermore, the action of $\operatorname{Spin}(n)$ on $\mathbb{R}^{n}$ is given in terms of multiplication in an algebra, $l_{n}$, containing $\operatorname{Spin}(n)$, and in which $\mathbb{R}^{n}$ is also embedded. It turns out that as a bonus, for $n \geq$ 3 , the group $\operatorname{Spin}(n)$ is topologically simpler than $S O(n)$, since $\operatorname{Spin}(n)$ is simply connected, but $S O(n)$ is not. By being astute, we can also construct a group, $\operatorname{Pin}(n)$, and a linear action of $\operatorname{Pin}(n)$ on $\mathbb{R}^{n}$ that induces a surjective homomorphism, $\rho: \operatorname{Pin}(n) \rightarrow$ $O(n)$, whose kernel is $\{-1,1\}$. The difficulty here is the presence of the negative sign in (2). We will see how Atiyah, Bott and Shapiro circumvent this problem by using a "twisted adjoint action," as opposed to the usual adjoint action (where $v \mapsto$ $\left.u v u^{-1}\right)$.

Definition (2-2) Given a field, $K$, a $K$-algebra is a $K$-vector space, $A$, together with a bilinear operation, $:: A \times A \rightarrow A$, called multiplication, which makes $A$ into a ring with unity, $I_{A}$. This means that is associative and that there is a multiplicative identity element, $1 I_{A}$ so that $I_{A} \cdot a=a \cdot I_{A}=a$, for all $a \in A$. Given two $K$-algebras $A$ and $B$, a $K$-algebra homomorphism, $h$ : $A \rightarrow B$, is a linear map that is also a ring homomorphism, with $h\left(I_{A}\right)=I_{B}$.

For example, the ring, $M n_{n}(K)$, of all $n \times n$ matrices over a field, $K$, is a $K$-algebra.

Tensor products( 2-3)

Let $E, F$ be two vector spaces over the field $\mathrm{K}(K=\mathbb{R}, \mathbb{C})$. Consider the (infinite) direct sum

$T(E, F)=\bigoplus_{(e, f) \in E \times F} K \quad(2.4)$

i.e., A tensor product of $E$ and $F$ is a pair,

$(E \otimes F, \otimes)$, where $E \otimes F$ is a $K$-vector space and $\otimes: E \times F \rightarrow$ $E \otimes F$ is a bilinear map.

Proposition.1. For any bilinear map $\phi: E \times F \rightarrow G$ there exists a unique linear

map $\Phi: E \otimes F \rightarrow G$ such that the diagram below is commutative. [15]

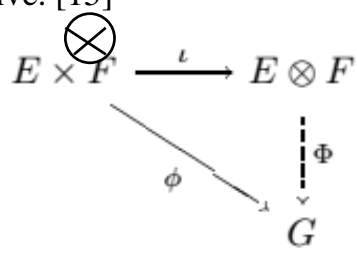

The vector space $E \otimes F$ is defined up to isomorphism. The vectors $\vec{u} \otimes \vec{v}$, where $\vec{u} \in E$ and $\vec{v} \in F$, generate $E \otimes F$.

CLIFFORD'S DESCRIPTION OF SPACE (2-4)

A Clifford geometric algebra $C \ell\left(\mathbb{R}^{n}\right)$ defines an associative real algebra over $n$ dimensions and in three dimensions $C \ell\left(\mathbb{R}^{3}\right)$ is eight-dimensional [14]. In this case we can adopt the three quantities $e_{1}, e_{2}, e_{3}$ for basis vectors that are defined to anticommute in the same way as Hamilton's quaternions, but unlike the quaternions these quantities square to positive one, that is $e_{1}^{2}=e_{2}^{2}=e_{3}^{2}=1$. Also, similar to quaternions, we

can combine scalars and the various algebraic components into a single number, called in this case a multivector

$$
\begin{gathered}
X=t+x_{1} e_{1}+x_{2} e_{2}+x_{3} e_{3}+n_{1} e_{2} e_{3}+n_{2} e_{3} e_{1}+n_{3} e_{1} e_{2} \\
+b e_{1} e_{2} e_{3}
\end{gathered}
$$

where $t, x_{1}, x_{2}, x_{3}, n_{1}, n_{2}, n_{3}, b \in \mathbb{R}$. Now define $j=e_{1} e_{2} e_{3}$ we find the relation $j e_{1}=e_{2} e_{3}$,

$j e_{2}=e_{3} e_{1}$ and $j e_{3}=e_{1} e_{2}$, which allows us to write

$X=t+\vec{x}+\vec{n} j+b j$

With vectors $\vec{x}=x_{1} e_{1}+x_{2} e_{2}+x_{3} e_{3}$ and $\vec{n}=n_{1} e_{1}+n_{2} e_{2}+$ $n_{3} e_{3}$

It can be shown that quaternions are isomorphic to the even subalgebra of the multivector, with the mapping $i \leftrightarrow e_{2} e_{3}$,

$j \leftrightarrow e_{1} e_{3}, k \leftrightarrow e_{1} e_{2}$ and the Gibbs vector can be replaced by the vector component of the multivector. Thus, within Clifford's system we can absorb the quaternion as

$q=t+j \vec{n}$ and a Gibbs vector $\vec{x}$ into a unified system.

We define Clifford conjugation on a multivector $X$ as $\bar{X}=t-\vec{x}-\vec{n} j+b j \quad(2.7)$

We define the amplitude squared of a multivector $X$ as $|X|^{2}=X \bar{X}$ that gives

$$
|X|^{2}=t^{2}-x^{2}+n^{2}-b^{2}+2 j(t b-\vec{x} \cdot \vec{n})
$$


Clifford conjugation that produces the multivector amplitude turns out to be the only viable definition for the metric as it is the only option that produces a commuting resultant and is thus an element of the center of the algebra. This is essential as we require the metric distances to be isotropic in space in order to be generally consistent with the principles of relativity. Once again we can observe the required invariant distance

$t^{2}-x^{2}$ appearing in the metric.

An important point to note for the multivector, is that in order to produce a meaningful metric, which consists of a combination of its various elements, then all of these components must be measured in the same units, as shown in Equation (8).

Now, beginning from 1983, the General Conference on Weights and Measures (CGPM) decided that the speed of light should be assumed constant and that a meter was then the distance traveled by light in a specified time interval equal to $1 / \mathrm{c} \mathrm{s}$ [15].The CGPM defines the speed of light as a universal constant $c=$ $299792458 \mathrm{~m} / \mathrm{s} \approx 3 \times 10^{8} \mathrm{~m} / \mathrm{s}$. Hence both time and distance are now measured in units of seconds, and it is therefore natural to adopt these units for all components of the multivector. Distances typically measured in meters therefore appear in the multivector with the conversion $\vec{x} \rightarrow \vec{x} / c$ and so are in units of seconds.

Interestingly, from the perspective of the multivector, $\mathrm{c}$ is simply taking the role of a units conversion factor, and so therefore the value of $c$ is obviously invariant between observers. Hence one confusion regarding time could arise due to the poor selection of units, as in order to properly relate space and time they should be measured in the same units, as now carried out by CGPM.

The role of the various terms in the metric can be understood from several perspectives. Geometrically they refer to the four geometric elements of three-dimensional space (points, lines, areas and volumes) combined in a natural way to form an invariant distance. These four geometric elements described algebraically by the scalar, vectors, bivectors and trivectors of Clifford geometric algebra. In terms of physical quantities they are commonlyreferred to as scalars, vectors, pseudo vectors and pseudo scalars. Now, scalars and vectors are well understood physically as quantities such as energy and momentum respectively. Pseudo vectors, also called axial vectors, can be understood physically as rotational quantities, such as angular momentum, torque and the magnetic field. Pseudo scalars are less commonly understood and describe the nature of magnetic monopoles or helical motion.

The multivector generalization of the quaternions now allows a full algebraic description of three-dimensional space, as the scalar, vector, bivectors and trivectors components now correspond directly with the geometrical quantities of points, lines, areas and volumes found in three dimensions.Additionally these four quantities describe the range of physical quantities described as scalars, vectors, pseudo vectors (or axial vectors) and pseudo scalars [13]. Now, similar to quaternions, as we also identify time as the scalar part of space, time is now imputed the geometrical meaning and topology of a scalar point-like quantity.

This can be contrasted with the linear description of space as vectors implied by Minkowski and so we can see now a sharp geometrical distinction between space and time when described within the multivector. We now wish to show briefly that the multivector provides a viable formalism to describe both classical and relativistic dynamics from which a definition of time can

\section{The Minkowski spacetime}

A few years later to Einstein's seminal paper of 1905 introduced a revolutionary new description of space and time , Minkowski showed that these phenomena could be simply interpreted as the geometrical properties of a four-dimensional space-time continuum subject to the metric

$d s^{2}=c^{2} d t^{2}-d \mathrm{x}^{2}$

$$
=c^{2} d t^{2}-d x_{1}^{2}-d x_{2}^{2}-d x_{3}^{2}
$$

Where

$d \mathrm{x}^{2}=d \mathrm{x} \cdot d \mathrm{x}=d x_{1}^{2}+d x_{2}^{2}+d x_{3}^{2}$

is the contribution from three spatial dimensions and $t$ is the time in this reference frame, where we have assumed units such that the speed of light $c=1$.

In the Minkowski formulation we can write a space-time event as the four vector $X=[t, \overrightarrow{\mathrm{v}}]^{T}$, where the corresponding event for other inertial frames is found through the Lorentz transformation $X^{\prime}=\Lambda X$ where

$\Lambda$ is a $4 \times 4$ real matrix, which preserves the metric distance in Eq. (3.1). We reiterate these elementary results to illustrate firstly the unified nature of space and time, and secondly, that the metric has a mixed signature, as shown in Eq. (3.1).

Now, due to the fundamental importance of Minkowski spacetime, a variety of arguments have been proposed over the years seeking to derive this spacetime structure from more elementary considerations, such as the consistency of Newton's First Law, spacetime isotropy with non-instantaneous interaction propagation [31,20], a probability distribution resulting from quantum fluctuations of the spacetime metric, the requirements for the existence of observed elementary particles and dynamics in the context of basic quantum mechanical considerations [5, 33, 34], implications of electric/magnetic reciprocity [25], and the effective ill-posed nature of the Einstein field equation unless the Minkowski metric holds [35].

We will adopt the formalism of Clifford geometric algebra as it has been used previously by several authors to duplicate the Minkowski spacetime structure. Two well-known implementations are

(i) spacetime algebra (STA) [22] and

(ii) the algebra of physical space (APS) [2]. The formalism of APS will be shown to be a special case of our approach detailed shortly, whereas in the case of STA the Minkowski metric is assumed axiomatically rather than deduced from the algebra.

We now begin with the Clifford algebra of three dimensions and produce the Minkowski metric as an emergent property of this algebraic structure. While recovering standard results we nevertheless generalize the representation of spacetime events as well as the group of Lorentz transformations. The metric is found to produce a suitable Lagrangian for relativistic processes that indicates possible extensions to the electromagnetic interaction.

As we see before the Clifford's geometric algebra (GA) over three dimensions as an appropriate algebraic representation. This can be expressed using an object called a Multivector

$M=t+\vec{x}+j \vec{n}+j \quad$ (3.2)

where $\vec{x}=x_{1} e_{1}+x_{2} e_{2}+x n_{3} e_{3}$ a vector $j \vec{n}=n_{1} e_{2} e_{3}+$ $n_{2} e_{1} e_{3}+n_{3} e_{1} e_{2}$ a bivector, and $j=e_{1} e_{2} e_{3}$ trivector with $t, b, x_{1}, x_{2}, x_{3}, n_{1}, n_{2}, n_{3}$ real scalars 
Definition 2.1 (Clifford conjugation). We define Clifford conjugation of a multivector $M$ as $\bar{M}=t-\vec{x}-j \vec{n}+j b$

Definition 2.2 (Multivector amplitude). We define the amplitude squared of a multivector $\mathrm{M}$ through Clifford conjugation as

$|M|^{2}=M \bar{M}=t^{2}-x^{2}+n^{2}-b^{2}+2 j(t b-\vec{x} \cdot \vec{n})(3.4)$

forming a complex-like number. So we can define the multivector amplitude as $|M|=\sqrt{|M|^{2}}$.

Note that we have used the conventional dot product $\vec{x}$. $\vec{n}=1 / 2(x n+n x)$ which can now be defined in terms of the algebraic product of Clifford vectors, The group transformations Definition 2.4 (Bilinear multivector transformation)

We define a general bilinear transformation on a multivector $M$ as

$M^{\prime}=Y M Z$,

where $M, Y, Z \in C \ell\left(\mathbb{R}^{3}\right)$.

We then find the transformed multivector amplitude

$\left|M^{\prime}\right|^{2}=Y M Z \overline{Y M Z}=Y M Z \bar{Z} \bar{M} \bar{Y}$

$$
=|Y|^{2}|Z|^{2}|M|^{2}
$$

where we have used the anti-involution property of Clifford conjugation and the commuting property of the amplitude.

Hence, provided we specify a unitary $|Y|^{2}|Z|^{2}=1$ for these transformations, then the amplitude $|M|$ will be invariant. Without loss of generality, it is then convenient to impose the condition $|Y|^{2}|Z|^{2}= \pm 1$ The transformation in Eq. (2.5) is then the most general bilinear transformation that preserves the multi-vector amplitude and so produces an invariant distance over the space. We will focus on the special case $|Y|^{2}|Z|^{2}=+1$ that describe transformations that are continuous with the identity.Now, using the power series expansion of the exponential function, a multivector $\mathrm{Y}$ can be written in an exponential form [22, 23]

$Y=e^{c+p+j q+j d}$

provided $|Y| \neq 0$, where $c, d \in \mathbb{R}$ and

$p, q \in \mathfrak{R}^{3}$. Therefore, we find

$Y \bar{Y}=e^{c+p+j q+j d} e^{c-p-j q+j d}$

$$
=e^{2 c+2 j d}
$$

Now writing $=e^{r+j s}$, with $r, s \in \mathbb{R}^{3}$, we finally produce the general transformation operation

$M^{\prime}=e^{p+j q} M e^{r+j s}$,

which will leave the multivector amplitude invariant. The four three-vectors $p, q, r, s$ illustrate that the transformation is specified by twelve real parameters, thus generalizing the conventional six dimensional Lorentz group, consisting of boosts and rotations.

Now, if we represent space by the multivector in Eq. (3.2), then simple rotations of this space are described by the special case of Eq. (3.9)

$M^{\prime}=e^{-j \omega / 2} M e^{j \omega / 2}$,

which will produce a rotation of $\theta=|\vec{\omega}|$ radians about the axis $\vec{\omega}$. It is convenient to have separate notation for the Pythago-rean length of a vector $\vec{\omega}$, given by the unbolded symbol

$\omega=|\vec{\omega}|=\sqrt{\omega_{1}^{2}+\omega_{2}^{2}+\omega_{3}^{2}}$.

A further special case of Eq. (2.9) is found by selecting the vector exponent, which will correspond to conventional Lorentz boosts.

That is $M^{\prime}=e^{-\phi \hat{v} / 2} M e^{\phi \hat{v} / 2}$

where $\phi$ is defined through $\tanh \phi=v$

where $v=|\vec{v}|$ and where $v$ is the relative velocity vector between the two observers.

We can rearrange $\tanh \phi=v$ to give $\cosh \phi=\gamma$ and $\sinh \phi=\gamma v$. Hence

$e^{-\phi \hat{v}}=\cosh \phi-\hat{v} \sinh \phi=\gamma(1-v)$,

where $\gamma=1 / \sqrt{1-v^{2}}$.

In this case the vector $\mathrm{v}$ is identified with the relative velocity vector between frames whereas for rotations it is identified with the rotation axis. These results consistent with the known result that the Lorentz group is a sub-manifold of the Pauli algebra [36]. If we now consider the effect of a conven-tional Lorentz boost on the generalized eight-dimensional spacetime coordinate

$$
M=t+\vec{x}_{\|}+\vec{x}_{\perp}+j \vec{n}_{\|}+j \vec{n}_{\perp}+j b,
$$

where we split the spatial coordinate into components perpendicular and parallel to the boost direction ^ $\mathrm{v}$. We then find from Eq. (2.11) that $M^{\prime}=t e^{-\hat{e} \phi}+\vec{x}_{\|} e^{-\hat{e} \phi}+\vec{x}_{\perp}+j \vec{n}_{\|} e^{-\hat{e} \phi}+j \vec{n}_{\perp}+j b e^{-\hat{e} \phi}$

$$
=\gamma\left(t-v \vec{x}_{\|}\right)+\gamma\left(\vec{x}_{\|}+v t\right)+j \gamma\left(\vec{n}_{\|}-v b\right)+j \gamma\left(b-v \vec{n}_{\|}\right)
$$

(3.12),

which now shows the transformation of the full multivector subject to the conventional Lorentz boost operation. We can see that the plane $j \vec{n}_{\|}$orthogonal to the boost direction $v$ is expanded by the $\gamma$ factor to $j \gamma \vec{n}_{\|}$.

This implies that the bivectors do not refer to quantities such as areas or angular momentum of extended body as the parallel components are in fact unchanged by such boosts - but must refer to other axial vector-type quantities such as spin or the magnetic field. In fact, the bivector and trivector components $j \vec{n}+j b=$ $j(b+\vec{n})$ are transformed the same as a four-vector therefore have the same transformational properties as the four-spin fourvector. We can also see that the conventional Lorentz boost transformation splits the multivector space into two four dimensional subspaces $\mathbb{R} \oplus \mathbb{R}^{3}$ and $\wedge^{2} \mathbb{R}^{3} \oplus \Lambda^{3} \mathbb{R}^{3}$ represented by $t+\vec{x}$ and $j \vec{n}+j b$ respectively, where $\wedge$ refers to the exterior algebra. The first four-vector $t+\vec{x}$ can be identified as conventional spacetime if we identify the scalar a with the time $t$ and the second four-vector $j \vec{n}+j b$ as four-spin. Thus Eq. (3.1) appears to describe a unified formulation of spacetime that includes spin. For example, with respect to the metric in Eq. (3.4), by identifying the scalar a with time $t$, and $n=0, b=0$, we obtain the required invariant spacetime interval $t^{2}-x^{2}$. The fact that the conventional boost operation, shown in Eq. (3.12), effectively splits the multivector into two independent four-vector spaces perhaps illustrates why the four-vector is effective, even though a unified treatment using the multivector is preferable Hence, we can write a general spacetime event $X$, in differential form, as

$$
d X=d t+d x+j d n+j d b,(2.13)
$$

providing a generalization to eight-dimensional events, where the special case $d X=d t+d x$ is isomorphic to the conventional four vector $d X=[d t, d x]^{T}$. Referring to Eq. (3.4) this therefore has an amplitude $|d X|^{2}=d t^{2}-d x^{2}+d n^{2}-d x b^{2}+$ $2 j(d t d b-d x \cdot d n)$.

(3.14)

In general, the interval is therefore a complex -like number .Now, for the special case for particles that have a rest frame, defined as $d x=0$, with a corresponding proper time $d \tau$, we have $d \tau^{2}=\left|d X_{0}\right|^{2}=d t_{0}^{2}+d n_{0}^{2}-d b_{0}^{2}+2 j d t_{0} d b_{0}$,

(2.15) where the zero in the subscripts denotes the rest frame. Now as $d \tau^{2}$ is real we therefore require $d t_{0} d b_{0}=0$, and as we assume $d t_{0}>0$ then we have $d b_{0}=0$. Hence we have in the rest frame

$$
d \tau^{2}=d t_{0}^{2}+d n_{0}^{2}=d t^{2}-d x^{2}+d n^{2}-d b^{2} .
$$


(2.16)

Note that as the imaginary term is now zero in the rest frame and the interval is invariant, then the imaginary component will also remain zero in the boosted frame. We can then write for the proper time $e^{j u} d \tau=d X_{0}=d t_{0}+j d n_{0}$, (3.17)

where $\vec{u}$ is a three-vector. This then returns $|d X|^{2}=$ $\left(e^{j u} d \tau\right)\left(e^{-j u} d \tau\right)=d \tau^{2}$, as required. Note that $e^{j u}=\cos u+$ $j \hat{u} \sin u$, where $u=\sqrt{u^{2}}$ and $\hat{u}=\vec{u} / u$. Thus the proper time becomes identified with the even subalgebra of $C \ell\left(\mathbb{R}^{3}\right)$, which can be shown to be isomorphic to the quaternions. A connection is now also made to quantum mechanics, as the Pauli spinors are also isomorphic to the quaternions. Additionally, under a relativistic boost we will populate the full eight-dimensional multivector that has been shown to be isomorphic with the eight-dimensional Dirac spinor describing spin- $1 / 2$ particles[4]. Comparing a primed rest frame with the boosted frame we therefore find

$d t^{\prime 2}+d n^{\prime 2}=d t^{2}-d x^{2}+d n^{2}-d b^{2}$.

(2.18)

Now, as we have seen, the two-subspaces of $d t+d x$ and $j d n+$ $j d b$ are disjoint

under boosts and so we have

$d t^{\prime 2}=d t^{2}-d x^{2}$ or

$d t^{\prime}=d t \sqrt{1-(d x / d t)^{2}}$

$=d t \sqrt{1-v^{2}}=d t / \gamma$ and so gives the usual time dilation factor $\gamma=1 / \sqrt{1-v^{2}}$, where $v=d x / d t$.

Also, $d t^{\prime 2}>0$ that implies $d x^{2}<d t^{2}$ and so we have $v<1$, thus setting an upper limit speed. Hence, despite the fact that time in the rest frame is quaternionic, nevertheless the amplitude of this time $\mathrm{d} \tau$ is real and so we will find that standard results are returned. However, this recognition of time as a quaternion is planned to be further explored in a subsequent paper.

Affine space of real dimension In the standard embedding we may "imagine" the Euclidean $\mathbb{R}^{3}$ embedded in $\mathbb{R}^{4}$ as an affine space parallel to the $(x, y, z, 0)^{T}$ space of $\mathbb{R}^{4}$.

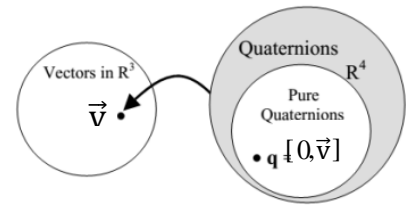

Fig.1 A one-to-one correspondence between pure quaternions and vectors in $\mathbb{R}^{3}[13]$

If this is too hard to imagine, then one can also proceed purely formally. In the standard embedding a point $(x, y, z) \in \mathbb{R}^{3}$ is represented by a four-Dimensional vector $(x, y, z, 1)^{T} \in \mathbb{R}^{4}$.

Nonzero scalar multiples are identified. The Infinite points are exactly those nonzero vectors of the form $(x, y, z, 0)^{T}$. They do not correspond to Euclidean points. Vectors of the form $(x, y, z, 0)^{T}$ may also be interpreted as homogeneous coordinates of the usual projective plane by ignoring the last entry.Thus we can literally say that $\mathbb{R P}^{3}$ consists of $\mathbb{R}^{3}$ (the homogeneous vectors $\left.(x, y, z, 1)^{T}\right)$ and a projective plane at infinity (the homogeneous vectors $(x, y, z, 0)^{T}$. We may also interpret this process inductively and consider the projective plane itself as composed of the Euclidean plane $\mathbb{R}^{2}$ (the vectors $(x, y, 1,0)^{T}$ )and a line at infinity (the vectors $\left.(x, y, 0,0)^{T}\right)$. The line may be considered a Euclidean line (the vectors $(x, 1,0,0)^{T}$ and finally a point at infinity (represented by $(1,0,0,0)^{T}$.

The objects dual to points in $\mathbb{R} \mathbb{P}^{3}$ will be planes. Similarly to points, planes will also be represented by four-dimensional vectors. In Euclidean terms we may consider the vector $(a, b, c, d)^{T}$ as representing the parameters that describe the affine plane

$\left\{(x, y, z) \in \mathbb{R}^{3} \mid a x+b y+c z+d=0\right\}$.

As usual, nonzero scalar multiples of the vector represent the same geometric object.If we interpret the equation in a setup of homogeneous coordinates, a point $(x, y, z, w)^{T}$ is incident to a plane $(a, b, c, d)^{T}$ if and only if $a x+b y+c z+d w=0$.

As in the two-dimensional setup, incidence is simply expressed by a scalar product being zero. The plane at infinity has homogeneous coordinates $(0,0,0,1)^{T}$. Finding a plane that passes through three given points

$p_{i}=\left(x_{i}, y_{i}, z_{i}, w_{i}\right), i=1,2,3$, thus translates to the task of solving a linear equation:

$\left[\begin{array}{llll}x_{1} & y_{1} & z_{1} & w_{1} \\ x_{2} & y_{2} & z_{2} & w_{2} \\ x_{3} & y_{3} & z_{3} & w_{3}\end{array}\right]\left[\begin{array}{l}a \\ b \\ c \\ d\end{array}\right]=\left[\begin{array}{l}0 \\ 0 \\ 0\end{array}\right]$.

In the next section we will deal with methods of performing this calculation explicitly. However, before we do so we will consider transformations in this homogeneous projective setup. The situation here is almost completely analogous to the two-D case. A transformation is represented by a simple matrix multiplication. This time we need a $4 \times 4$ matrix. The cases of usual affine transformations in $\mathbb{R}^{3}$ are again covered by special transformation matrices in which certain entries are zero. The following matrices represent a linear transformation of $\mathbb{R}^{3}$ with matrix A, a pure translation, a General affine transformation, and a general projective transformation, respectively:

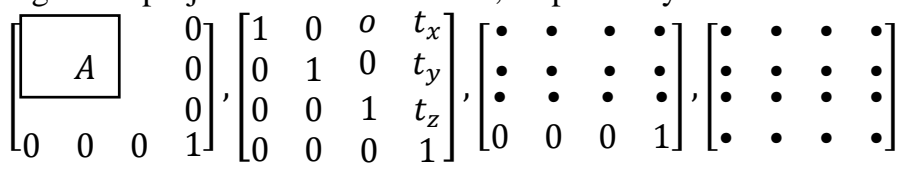

A "ø" stands for an arbitrary number. In every case we must assume that the determinant of the matrix is nonzero.Such a transformation $\mathrm{T}$ maps a point $\mathrm{p}$ to the associated image point. The correspond-ing transformation that applies to the homo-geneous coordinates of a plane is (as in the two-dimensional case) given by $\left(T^{-1}\right)^{T}$. By this choice incidence of points and planes is preserved by projective transformations:

$\langle p, h\rangle=0 \Leftrightarrow\left\langle T_{p},\left(T^{-1}\right)^{T} h\right\rangle=0$.

Instead of the transposed inverse $\left(T^{-1}\right)^{T}$ it is also possible to use the transposed adjoint $\left(T^{\Delta}\right)^{T}$, since they differ by only a scalar factor.Remark 12.1. A word of caution: One should consider the setup of presenting points and planes by four-dimensional vectors and expressing coincidence by the standard scalar product as a kind of interim solution that will be replaced by something more powerful later on. The problem that we will have to face soon is that lines will be represented by six-dimensional vectors and we have to create a notational system that handles points, lines, and planes in a unified way. For this we will have to give up the 
concept of indexing a vector entry with the position where it is placed in a vector.

\section{CONCLUSION}

Minkowski space is a vector space or affine space of real dimension non which there is an inner product or pseudoRiemannian metric of signature $(n-1,1)$, i.e., in the above terminology, $n-1$ "pluses" and one "minus".The Poincaré groupis the group of all isometries of Minkowski spacetime including boosts, rotations, and translations. The Lorentz groupis the subgroup of iso-Metries which leave the origin fixed and includes the boosts and rotations; members of this subgroup are called Lorentz transfor-mations. Among the simplest Lorentztransformations is a Lorentz boost.

The archetypal Lorentz boost is[2]

$$
\left[\begin{array}{l}
M_{0}^{\prime} \\
M_{1}^{\prime} \\
M_{2}^{\prime} \\
M_{3}^{\prime}
\end{array}\right]=\left[\begin{array}{cccc}
\gamma & -\beta \gamma & 0 & 0 \\
-\beta \gamma & \gamma & 0 & 0 \\
0 & 0 & 1 & 0 \\
0 & 0 & 0 & 1
\end{array}\right]\left[\begin{array}{l}
M_{0} \\
M_{1} \\
M_{2} \\
M_{3}
\end{array}\right]
$$

where $\gamma=\frac{1}{\sqrt{1-\frac{v^{2}}{c^{2}}}}$ is the Lorentz factor, and $\beta=\frac{v}{c}$.

All four-vectorsin Minkowski space transform according to the same formula under Lorentz transformations.Minkowski diagramsillustrate Lorentz transformations.[2]

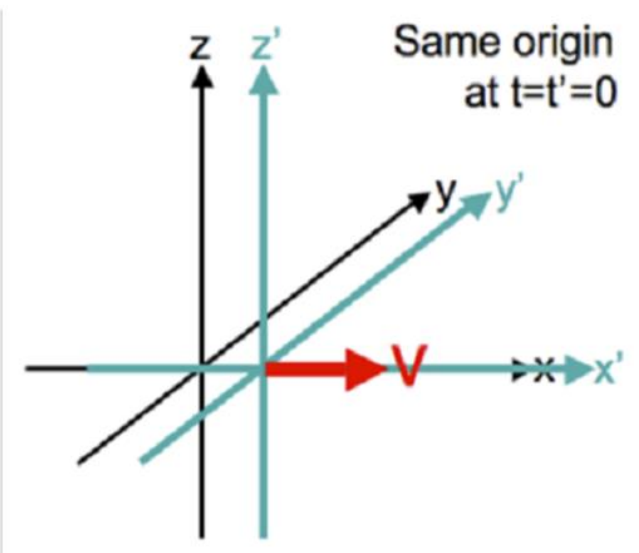

Fig .2Standard configuration of coordinate systems for Lorentz transformations.

\section{REFERENCES}

[2] [2]http://en.wikipedia.org/wiki/Minkowski-space

[3] [3]James M.Chappel1* and others ,Time As Geometric Property of Space, Dumitru Baleanu, University of Craiova, Romania , 17 November 2016

[4] [4] Harvey Reall, Part 3 General Relativity,4/11/2013

[5] [5] Sean M. Carroll, Lecture Notes on General Relativity, nstitute for Theoretical Physics University of California Santa Barbara, CA 93106 December 1997

[6] [6] David Hestenes, Spacetime Physics with Geometric Algebra, Department of Physics and Astronomy Arizona State University, Tempe, Arizona 852871504 June 2003.

[7] [7] James M. Chappell and others, Exploring the origin of Minkowski Spacetime,arXiv:1501.04857v4[physics.gen-ph] , 6 Aug 2016

[8]

[9] [9] Jean Gallier, Clifford Algebras, Clifford Groups, and a Generalization of the Quaternions:The Pin and Spin Groups, Department of Computer and Information Science University of Pennsylvania Philadelphia, PA 19104, USAJanuary 31, 2012

[10] [10] Bernhard Rothenstein1(Politehnica University of Timisoara, Physics Department)and Stefan Popescu2(Siemens AG, Erlangen, Germany) Lorentz transformations: Einstein's derivation simplified, without date

[11] [11] J"urgen Richter-Gebert, Perspectives on Projective Geometry , (Draft Version)October 3, 2010 Springer

[12] [12] Darryl D Holm, Geometric MechanicsPart II: Rotating, Translating and Rolling, Mathematics Department Imperial College London 2nd Edition 23 Sept 2011

[13] [13] R.Mukunda , Quaternions: From Classical Mechanics to Computer Graphics, and Beyond, University of Canterbury Christchurch, New Zealand, Conference in Mathematics 2002. Invited Paper.

\section{AUTHORS}

First Author - Emran Sasi Althabit*, PH.D of mathematics, SUDAN UNIVERISITY SEEINCE AND TECHNOLOGY (Khartoum), +218926083695 omransasi045@gmail.com Second Author - Ismail Mustafa Mohamed**, PH.D in mathematics, AL NEELAN UNIVERISITY (Khartoum), +249124699207 abuahd91@gmail.com 